\title{
Unilateral corneal leukoplakia without limbal involvement
}

This article was published in the following Dove Press journal:

International Medical Case Reports Journal

18 May 2015

Number of times this article has been viewed

Koji Hirano'

Mihoko Koide ${ }^{2}$

Yoshikazu Mizoguchi ${ }^{3}$

Yasuhiro Osakabe ${ }^{4}$

Kaoru-Araki Sasaki ${ }^{5}$

'Department of Ophthalmology, Ban Buntane Hotokukai Hospital, School of Medicine, Fujita Health University, Nagoya, Japan; ${ }^{2}$ Koide Internal Medicine and Eye Clinic, Nagoya, Japan; ${ }^{3}$ Department of Pathology, Ban Buntane Hotokukai Hospital, School of Medicine, Fujita Health University, Nagoya, Japan; ${ }^{4}$ Department of Molecular Pathology, Tokyo Medical University, Tokyo, Japan; ${ }^{5}$ Department of Ophthalmology, Japan Health Care Organization, Hoshigaoka Medical Center, Hirakata, Japan
Correspondence: Koji Hirano

Department of Ophthalmology, Ban Buntane Hotokukai Hospital, School of Medicine, Fujita Health University, 3-6-10, Otoubashi, Nakagawa-ku, Nagoya 454-8509, Japan

$\mathrm{Tel}+8 \mid 5232$ | $817 \mid$

Fax +8I 523224734

Email kojihira@fujita-hu.ac.jp
Purpose: Leukoplakia is the term given to a white patch or plaque that is found mainly on the oral mucus membrane. It can occasionally be seen on the corneal surface. We report our clinical and histopathological findings in a case of unilateral corneal leukoplakia.

Methods: A 26-year-old woman was referred to our hospital because of a white patch on her right cornea that continued to expand. She first noticed the white patch when she was 20 years old, and the white patch had expanded to cover the pupillary area affecting her vision. After plastic surgery on both eyelids for bilateral entropion to alleviate the pain caused by the eyelashes rubbing the cornea, the white corneal patch decreased in size. Because of this reduction, we performed surgery to remove the patch with microforceps under topical anesthesia. The plaque was removed easily and completely, and submitted for histopathological examination.

Results: Histopathological examination showed that the specimen had characteristics of epidermis with a basal cell layer, spinous cell layer, granular cell layer, and horny layer with hyperkeratosis. She was diagnosed with leukoplakia of the corneal surface. The basic structure of the squamous cell layer was preserved, and there were no signs of metaplasia. Six months after the removal of the leukoplakia, no recurrence was seen and her corrected decimal visual acuity recovered to 1.0 .

Conclusion: Our case of unilateral corneal leukoplakia without limbal involvement was most likely caused by chronic irritation of the cornea by the eyelashes. Although it was totally removed with good recovery of vision, we continue to follow the patient because of the potential of malignant transformation.

Keywords: corneal leukoplakia, hyperkeratosis, eyelashes, chronic friction

\section{Introduction}

Ophthalmologists occasionally encounter a patient with an opaque corneal epithelial lesion causing a reduction of vision. These lesions can be due to a neoplasm, ${ }^{1}$ epithelial dysplasia, ${ }^{2}$ a benign hyperplastic reaction, ${ }^{3,4}$ dystrophies and degenerations, or plaque formation. ${ }^{5}$ Various irritants (eg, ultraviolet exposure, foreign bodies, and microorganisms) can affect the corneal surface and be the cause of various kinds of pathologies.

Leukoplakia is a prediagnostic term and is defined as a white patch or plaque that cannot be characterized clinically or pathologically as any other disease (World Health Organization 1978). ${ }^{6}$ The plaque can cover the mucus membrane of the oral cavity, vocal cords, gastrointestinal tract, or mucosal surface of the urinary tract and genitals. Because the term leukoplakia describes a clinical appearance, it has a wide range of histopathologic appearances. However, it generally refers to an epidermal thickening or hyperkeratosis. ${ }^{7}$ 
We examined a patient with unilateral corneal leukoplakia that resembled a calcium deposit or the mucus plaques of vernal keratoconjunctivitis. We present our findings and successful treatment of the corneal leukoplakia.

\section{Materials and methods}

A 26-year-old young woman was referred to one of the authors (Koji Hirano) because of an irregularly-shaped whitish patch on the cornea of her right eye that resembled a calcium deposit or the mucus plaque of vernal keratoconjunctivitis. She had entropion of both eyelids, and to avoid pain due to the scraping of the eyelashes, she began wearing cosmetic, tinted soft contact lenses from the age of 18 years. She had noticed a white corneal deposit on her right eye at the age of 20 years and had worn the tinted contact lenses to conceal the opaque cornea. Although the contact lenses prevented the irritation of the corneas by her eyelashes while she was wearing them, the irritation occurred every day after she removed the lenses. As the corneal opaque lesion grew larger, she noticed a decrease in the vision in her right eye. She was known to have perennial allergic conjunctivitis and rhinitis, but she did not have any history of other allergic problems, including atopic dermatitis. She also did not have any family history of corneal disorders. She was then referred to our clinic for a more detailed examination of the white corneal plaque.

Our initial examination showed that her decimal bestcorrected visual acuity was 0.1 (noncorrectable) OD, and
0.5 (with $1.0 \times-1.5 \mathrm{D}=\mathrm{Cyl}-1.0 \mathrm{D} \mathrm{Ax} 30^{\circ}$ ) OS. She was noted to have entropion of the upper and lower eyelids bilaterally, and the eyelashes were seen to touch the corneal surface whenever she blinked (Figure 1A and B). A discrete whitish patch or plaque was observed on the right corneal surface, which was associated with an invasion of blood vessels from the limbus (Figure 1C). Fluorescein staining revealed punctate epithelial defects around the whitish plaque, and the tear film was shed on the surface of the plaque (Figure 1D). Despite a careful slit-lamp biomicroscopic examination, we could not detect any pathological findings in the left cornea.

Although Propionibacterium spp. was detected by extended cultures of samples scraped from the lesion, it did not seem to be significantly associated with the lesion. We decided to follow her without treatment but scheduled an examination every 2 months.

Four months from the first visit, no remarkable changes were observed, and because of her complaint of bilateral foreign body sensation caused by the eyelashes, entropion surgery was performed on the upper and lower eyelids of both eyes by one of the authors (Mihoko Koide).

Seven weeks after the plastic surgery on the right eye, the whitish plaque was smaller (Figure $2 \mathrm{~A}$ and B), and her visual acuity was improved to 0.3 (with $0.7 \times-3.0 \mathrm{D}$ ). Encouraged by the fact that the lesion was reduced, we performed surgery with microforceps to remove the plaque, under topical anesthesia. The whitish plaque was removed

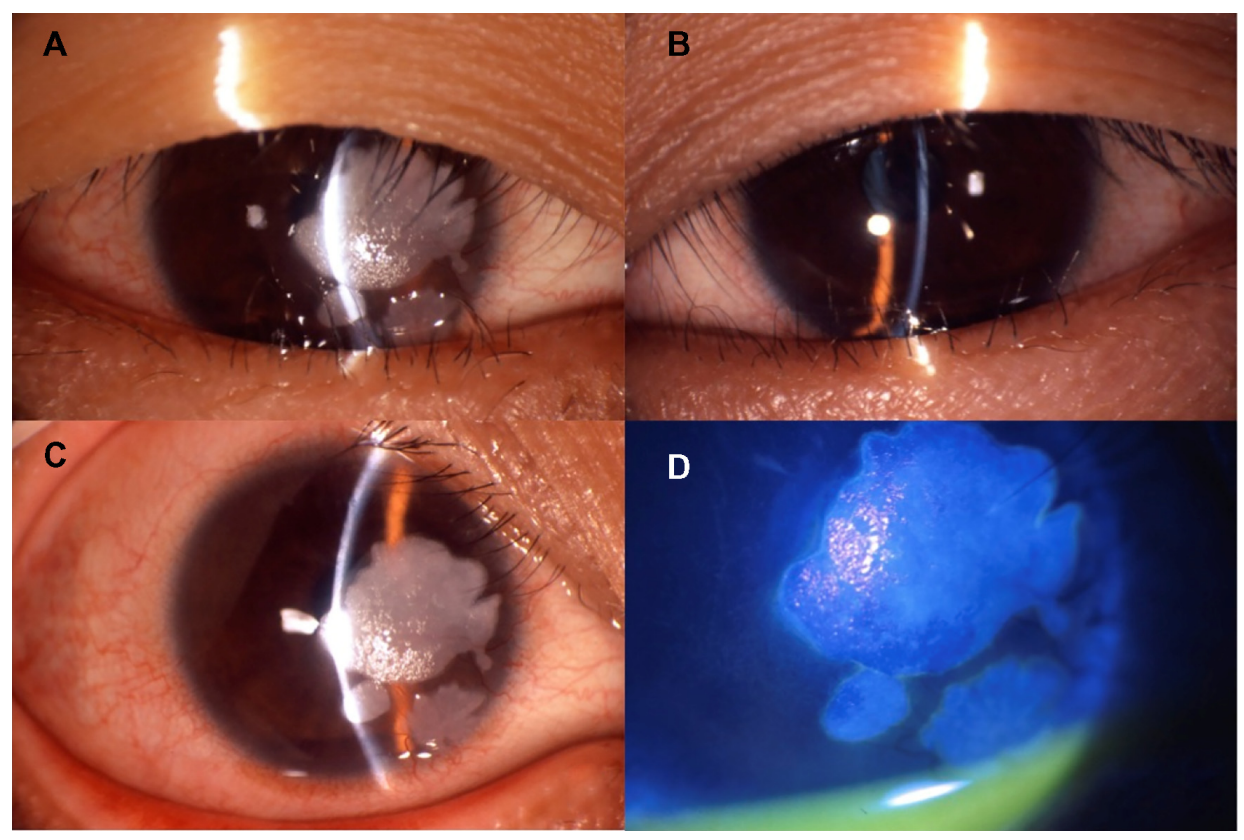

Figure I Findings in a patient with a white corneal plaque on the right eye at initial examination.

Notes: Entropion is present bilaterally and the eyelashes from both the upper and lower lids can be seen touching the cornea (A, right eye; $\mathbf{B}$, left eye). The white patches are limited to the corneal surface $(\mathbf{C})$. Fluorescein is shed on the surface of these patches, and superficial punctate epithelial defects are seen surrounding the lesion (D). 

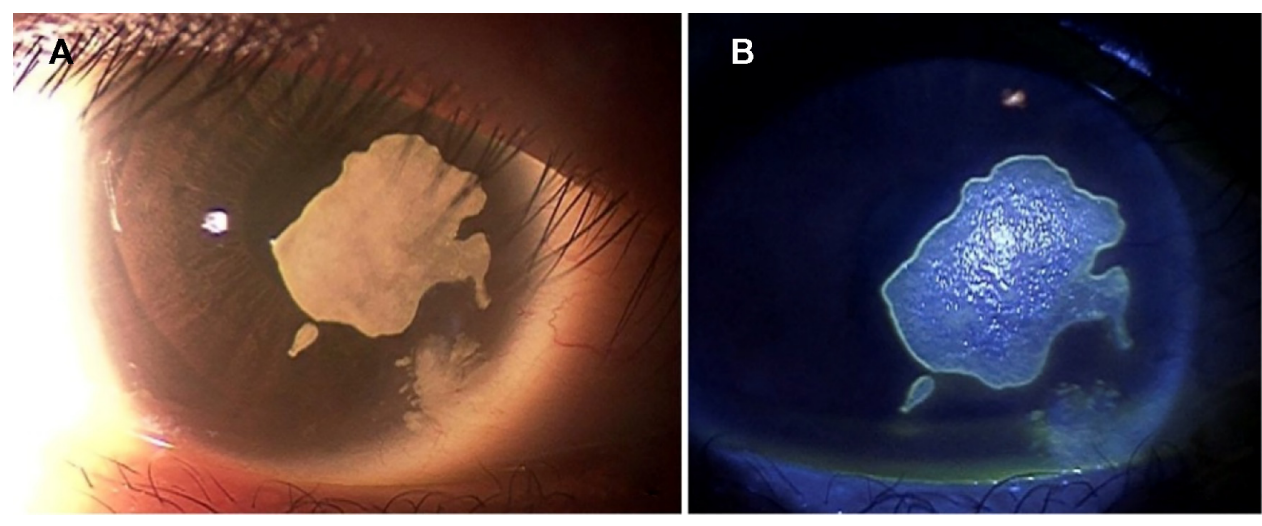

Figure 2 Findings 7 weeks after plastic surgery of the right eyelid (6 months after first visit).

Note: There is a decrease in the size of the white patches $(\mathbf{A})$, and the hyperfluorescence surrounding the lesion indicates that the edge is lifted up (B).

easily, and the underlying tissue appeared unaffected under the surgical microscope. The removed plaque was submitted for histopathological examinations.

An informed consent was obtained from the patient for the reporting of the clinical course and the pathological findings of the removed lesion.

\section{Results}

There were no signs of any recurrences, for at least 6 months after the removal of the white plaque. The cornea remained transparent except for ghost vessels. Her corrected decimal visual acuity improved to 1.0. Histopathological examination of the incised white plaque showed it had characteristics of the epidermis, viz basal cell layer in the area close to the epithelium or Bowman's layer of the cornea, a spinous cell layer, a granular cell layer on it, and a horny layer on the surface (Figure 3). Although the horny layer appeared to be hyperkeratotic, the basic structure of the squamous cell layer was preserved and there were no signs of metaplasia. Microorganisms were not detected in the PAS (Periodic acid-Schiff) stained samples, and inflammatory cells, such as leukocytes and lymphocytes, were not observed.

\section{Discussion}

Histopathologically, the white plaque consisted of an epidermal thickening associated with hyperkeratosis which is characteristic of leukoplakias. ${ }^{7}$ Leukoplakia is considered to be mainly a premalignant change of oral mucosa, and its presence increases the risk of malignant transformation by about 100 times over clinically normal oral mucosa. ${ }^{6}$

The etiology of leukoplakia is unknown; however, for the oral mucosa, the use of tobacco, alcohol abuse, irritating foods, and chronic friction, as from ill-fitting dentures or jagged teeth, are reported to have some associations with the development of leukoplakia. ${ }^{7}$ More recently, infections by the human papillomavirus (HPV) have been considered to have a causal relationship with leukoplakia. ${ }^{8} \mathrm{We}$ did not perform any immunohistochemical studies for the detection of HPV, and therefore cannot rule out HPV infection.

Our case had a chronic irritation of the cornea by the eyelashes due to entropion of both upper and lower eyelids. Because the size of the white patch decreased after the entropion surgery, the chronic irritation by the eyelashes was most likely the cause and progression of the leukoplakia. The continuous irritation by the eyelashes may have induced the corneal epithelial cells and stromal cells to secrete some extra-ordinal proteins, ${ }^{9}$ and it is also possible that the differentiation of corneal epithelial cells to epidermal cells may have been induced by the trichiasis.

The incidence of leukoplakia on the ocular surface area is unknown. According to Duke-Elder and Leigh, leukoplakia is a relatively common phenomenon, and it appears on

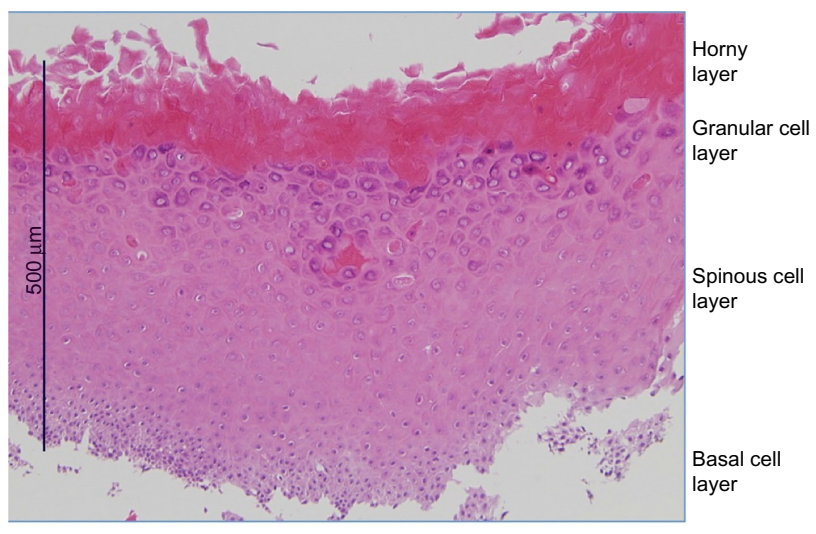

Figure 3 Histopathological finding of the white patch excised from the cornea. Notes: Ectopic epidermis with basal cell layer (basal), spinous cell layer (spinous), granular cell layer (granular), and horny layer (horny) on the surface can be seen. Uniform, thick layer horny indicates hyperkeratosis. The basic structure of squamous cell layer is preserved. 
the limbus and tends to spread over cornea and bulbar conjunctiva. On the other hand, a corneal leukoplakia without limbal involvement is rare. ${ }^{10}$ However, it is relatively rare to encounter this pathological phenomenon even with the limbal involvement. Because leukoplakia, before the definition of World Health Organization in 1978, can have different pathological associations such as neoplasia, dysplasia, and benign hyperplastic reactions, it might have been more common on the ocular surface at the time of the publication of Duke-Elder and Leigh's text. Another possibility is the marked decrease of trachoma, which Duke-Elder and Leigh listed a cause of chronic inflammation in the anterior segment of the eye. ${ }^{10}$

It is still not clear why the leukoplakia occurred unilaterally. The entropion was present in both eyelids and the irritation of the cornea by the eyelashes was bilateral, and yet the leukoplakia developed in only one eye.

Although the leukoplakia did not recur for at least 1 year after it was peeled off, we continue to follow this patient because the leukoplakia can be a premalignant condition.

In conclusion, our case of a unilateral corneal white patch was histopathologically diagnosed as leukoplakia, and was most likely caused by chronic irritation by the eyelashes. Although it was totally removed without difficulty and with good recovery of vision, we continue to follow the patient because of the potential of malignant transformation.

\section{Disclosure}

The authors report no conflicts of interest in this work.

\section{References}

1. Basti S, Macsai MS. Ocular surface squamous neoplasia. A review. Cornea. 2003;22(7):687-704.

2. Busin M, Spitznas M, Brauweiler P. Leukoplakia of the cornea presenting as fingerprint epithelial lines: a case report. Int Ophthalmol. 1991;15: 299-301.

3. Fatima A, Matalia HP, Vemuganti GK, Honavar SG, Sangwan VS. Pseudoepitheliomatous hyperplasia mimicking ocular surface squamous neoplasia following cultivated limbal epithelium transplantation. Clin Exp Ophthalmol. 2006;34:889-891.

4. Finger PT, Chin KJ, Wong JJ, Iacob CE. Reactive keratoma of the central corneal epithelium. Eur J Ophthalmol. 2009;19(3):484-486.

5. Donshik PC, Ehlers WH. Clinical immunologic diseases. In: Smolin G, Thoft RA, editors. The Cornea. Scientific Foundations and Clinical Practice. 3rd ed. Boston, MA: Little, Brown and Company; 1994: 354-356.

6. Morgan PR. Neoplasms and precancerous conditions of the oral mucosa. In: McGee JO, Isaacson PG, Write NA, editors. Oxford Textbook of Pathology: Volume 2a: Pathology of Systems. Oxford, UK: Oxford University Press; 1992:1063.

7. Kumar V, Abbas AK, Fausto N, Mitchel RN. Leukoplakia and erythroplakia. In: Kumar V, Abbas AK, Fausto N, Mitchel RN, editors. Robbins Basic Pathology. 8th ed. Philadelphia, PA; Saunders; 2007: 581-582.

8. Feller L, Lemmer J. Oral leukoplakia as it relates to HPV infection: a review. Int J Dent. 2012;2012:540561. doi: 10. 1155/2012/540561.

9. Araki-Sasaki K, Nakamura M, Kitagawa K, et al. Lactoferrin Glu561Asp facilitates secondary amyloidosis in the cornea. $\mathrm{Br} J$ Ophthalmol. 2005;89:684-688.

10. Duke-Elder S, Leigh AG. Cysts and tumors. In: Sir Duke-Elder, editor. System of Ophthalmology, VIII. Disease of the Outer Eye. London, UK: Henry Kimpton Publishers; 1965:1146-1153.
International Medical Case Reports Journal

\section{Publish your work in this journal}

The International Medical Case Reports Journal is an international, peer-reviewed open-access journal publishing original case reports from all medical specialties. Previously unpublished medical posters are also accepted relating to any area of clinical or preclinical science. Submissions should not normally exceed 2,000 words or
Dovepress

4 published pages including figures, diagrams and references. The manuscript management system is completely online and includes a very quick and fair peer-review system, which is all easy to use. Visit http://www.dovepress.com/testimonials.php to read real quotes from published authors. 